\title{
Three novel mutations in the ACTA2 gene in German patients with thoracic aortic aneurysms and dissections
}

\author{
Sabine Hoffjan ${ }^{\star 1,5}$, Stephan Waldmüller ${ }^{2,5}$, Wulf Blankenfeldt ${ }^{3}$, Judith Kötting ${ }^{1}$, Petra Gehle ${ }^{4}$, Priska Binner ${ }^{2}$, \\ Joerg T Epplen ${ }^{1}$ and Thomas Scheffold ${ }^{2}$
}

\begin{abstract}
Mutations in the gene encoding smooth muscle cell alpha actin (ACTA2) have recently been shown to cause familial thoracic aortic aneurysms leading to type A dissections (TAAD) and predispose to premature stroke and coronary artery disease. In order to further explore the role of $A C T A 2$ variations in the pathogenesis of TAAD, we sequenced the coding regions of this gene in 40 unrelated German patients with TAAD (with $(n=21)$ or without $(n=19)$ clinical features suggestive of Marfan syndrome). All patients had previously tested negative for mutations in the FBN1 and TGFBR2 genes. We identified three novel ACTA2 mutations and mapped them on a three-dimensional model of actin. Two mutations affect residues within (M49V) or adjacent to (R39C), the DNAse-I-binding loop within subdomain 2 of alpha actin. They were observed in families with recurrent aortic aneurysm (R39C) or aortic dissection (M49V). The third mutation causes an exchange in the vicinity of the ATP-binding site (G304R) in a patient thought to have isolated TAAD. None of the affected individuals had clinical features typical for Marfan syndrome, and no case of premature stroke or coronary artery disease was reported from the affected families. In conclusion, we underscore the role of ACTA2 mutations in nonsyndromic TAAD and suggest that ACTA2 should be included in the genes routinely investigated for syndromic and nonsyndromic TAAD. Detailed clinical investigations of additional families are warranted to further explore the full range of phenotypic signs associated with the three novel mutations described here. European Journal of Human Genetics (2011) 19, 520-524; doi:10.1038/ejhg.2010.239; published online 19 January 2011
\end{abstract}

Keywords: ACTA2; marfan syndrome; TAAD; aneurysm; dissection; actin

\section{INTRODUCTION}

Thoracic aortic aneurysms leading to type A dissections (TAAD) can result from inherited connective tissue disorders such as Marfan syndrome (MFS), Ehlers-Danlos syndrome or Loeys-Dietz syndrome caused by mutations in the FBN1, COL3A1, TGFBR2 and TGFBR1 genes, respectively. In addition, familial aggregation studies revealed that a significant proportion of nonsyndromic TAAD also has a familial background and appears to be inherited in an autosomal dominant manner. ${ }^{1}$ Recently, mutations in two genes encoding smooth muscle cell (SMC) contractile proteins were identified in families with TAAD: the gene encoding SMC $\alpha$-actin (ACTA2) on chromosome 10q22-24 $4^{2}$ and the gene encoding the $\beta$-myosin heavy chain (MYH11) on chromosome 16p13. ${ }^{3}$ The encoded proteins are involved in SMC contraction through ATP-fuelled cyclic interaction of the myosin motor with actin filaments. ${ }^{4}$ Similar to the situation found in hypertrophic and dilated cardiomyopathy, mutations of the vascular isoforms of actin and myosin are thought to disturb SMC contractile function, thereby triggering profound remodelling of the vessel wall, followed by an increase in aortic stiffness and, ultimately, aortic dilation and dissection. , $3,5,6$ It is worth noting that MYH11 mutations appear to be rare (only five mutations reported so far) and have been exclusively found in patients displaying a syndrome comprising of TAAD and patent ductus arteriosus, a common congenital cardiovascular malformation. ${ }^{7}$ In contrast, ACTA2 mutations were found to be responsible for approximately $14 \%$ of familial TAAD and are believed to interfere with the normal assembly of actin filaments. ${ }^{2}$ The resulting vascular phenotype includes SMC hyperplasia and disarray, and is therefore reminiscent of the histopathological picture seen in carriers of mutations in the MYH11 gene. ${ }^{5}$ It has been claimed that in addition to TAAD, ACTA2 mutations predispose patients to a variety of vascular diseases including premature coronary artery disease $(\mathrm{CAD})$ and premature ischaemic strokes. ${ }^{6}$

In order to further explore the role of ACTA2 variations in the pathogenesis of TAAD, we sequenced the coding regions of this gene in 40 unrelated German patients with TAAD who had previously tested negative for mutations in the FBN1 and TGFBR2 genes.

\section{SUBJECTS AND METHODS}

Subjects

A total of 40 unrelated German patients with TAAD were recruited through the Department of Human Genetics, Ruhr-University Bochum, Germany, and the Institute for Heart and Circulation Research, University of Witten/Herdecke, Germany. All probands had been enroled initially with suspected MFS because of thoracic aortic disease at young age (mostly under the age of 50 years).

\footnotetext{
${ }^{1}$ Department of Human Genetics, Ruhr-University Bochum, Bochum, Germany; ${ }^{2}$ Institute for Heart and Circulation Research, University of Witten/Herdecke, Dortmund, Germany; ${ }^{3}$ Max Planck Institute of Molecular Physiology, Department of Physical Biochemistry, Dortmund, Germany; ${ }^{4}$ Department of Cardiothoracic and Vascular Surgery, Deutsches Herzzentrum Berlin, Berlin, Germany

*Correspondence: Dr S Hoffjan, Department of Human Genetics, Ruhr-University Bochum, Universitätsstrasse 150, 44801 Bochum, Germany. Tel: +49 234 32 23823; Fax: +49 23432 14196; E-mail: sabine.hoffjan@rub.de

${ }^{5}$ These authors contributed equally to this work.

Received 6 September 2010; revised 1 December 2010; accepted 1 December 2010; published online 19 January 2011
} 
In detail, 21 patients showed additional clinical features suggestive of MFS but did not fulfil the clinical criteria for this hereditary connective tissue disease, whereas the remaining 19 patients were thought to have TAAD without additional MFS features. Molecular genetic analysis of the FBN1 and TGFBR2 genes had been previously performed and did not reveal a causative mutation in any of the probands. All participants were Germans of European origin. Written informed consent was obtained from all subjects. The study was approved by the Ethics Committees of the Universities of Bochum and Witten/Herdecke, and the Declaration of Helsinki protocols were followed.

\section{Mutation detection}

DNA was extracted from peripheral blood according to a standard method. ${ }^{8}$ In all 40 patients, the 9 exons of the ACTA2 gene were amplified by PCR using primers specific to exon-flanking intronic sequences, and both strands of the DNA were sequenced using the Beckman platform as previously described. ${ }^{9}$ The exact PCR and sequencing conditions, as well as the primer sequences, are available upon request. The mutation nomenclature used follows the recommendations of the Human Genome Variation Society (HGVS) and refers to the GenBank reference sequences NM_001613.2 (mRNA) and NP_001604.1 (protein). Exons affected by possibly deleterious mutations were sequenced in 96 healthy control individuals, in order to confirm that these variants are not frequent polymorphisms. Online amino-acid substitution prediction programs used included PolyPhen and Panther.

\section{Protein modelling}

The protein model of rabbit $\alpha$-actin (1J6Z) ${ }^{10}$ was derived from the RCBS Protein Data Bank ${ }^{11}$. The ribbon plots were drawn using the program ViewerPro version 4.2 (Accelrys Inc., San Diego, CA, USA) and PyMOL Molecular Graphics System (Delano Scientific, San Carlos, CA, USA) ${ }^{12}$.

\section{RESULTS}

Detection of three novel mutations in the ACTA2 gene

We identified novel ACTA2 mutations in 3 of the 40 analysed patients with TAAD. Two mutations were found in families with recurrent aortic aneurysm (family 1) or aortic dissection (family 2). The third mutation was found in a patient thought to have isolated TAAD (family 3; see below for a detailed description of the clinical findings in these families). None of the 21 individuals with clinical features suggestive of MFS was found to carry a possibly disease-causing mutation in ACTA2. Among the remaining 19 patients, no significant clinical differences were observed between the three individuals carrying ACTA2 mutations and the nonmutated patients. The three mutations c.115C $>\mathrm{T}$ (p.R39C) in exon 2, c.145A $>\mathrm{G}$ (p.M49V) in exon 3 and c.910G >C (p.G304R) in exon 8 of the ACTA2 gene, all are heterozygous missense mutations that affect evolutionarily conserved amino acids (Figure 1), one of which, Arg 39, has already been implicated in TAAD (Table 1). It is worth noting that the second mutation, M49V, has previously been described for the skeletal isoform of $\alpha$-actin (encoded by the gene ACTA1) in patients with nemaline or cap-type congenital myopathy. ${ }^{13,14}$ All three mutations were absent from 192 control chromosomes and were not listed in the GenBank dbSNP library. The mutation R39C was predicted to have benign consequences by the PolyPhen program, but it was called 'deleterious' by the program PANTHER. Both programs predicted a damaging effect of the two other mutations, M49V and G304R. Taken together, the findings suggest that these mutations are the primary cause of TAAD in the investigated families.

\section{Clinical findings}

Family 1 (p.R39C). The mutation was initially identified in a 38year-old female proband (patient III.5, Figure 2) suffering from mild aortic dilation and insufficiency. She had undergone two uneventful pregnancies and deliveries, and appeared physically stable. One of her brothers (patient III.2) had developed an aneurysm of the ascending aorta (that extended to the abdominal aorta) at the age of 25 years and had died at the age of 46 years in the course of a consecutive thoracic operation. No DNA sample was available from this individual to confirm the suspected presence of the ACTA2 mutation. The second brother (patient III.1) was diagnosed with a thoracic aortic aneurysm at the age of 40 years (aortic root diameter $57 \mathrm{~mm}$ ), and the 44-yearold sister (patient III.4) also showed mild aortic dilation and insufficiency (aortic root diameter $36 \mathrm{~mm}$ ). In these two siblings, the R39C mutation was confirmed, as well as in her father who was diagnosed with a thoracic aortic aneurysm at the age of 69 years (patient II.1). The grandfather (patient I.1) was reported to have died suddenly at an early age, but no further information was available. The 47-year-old

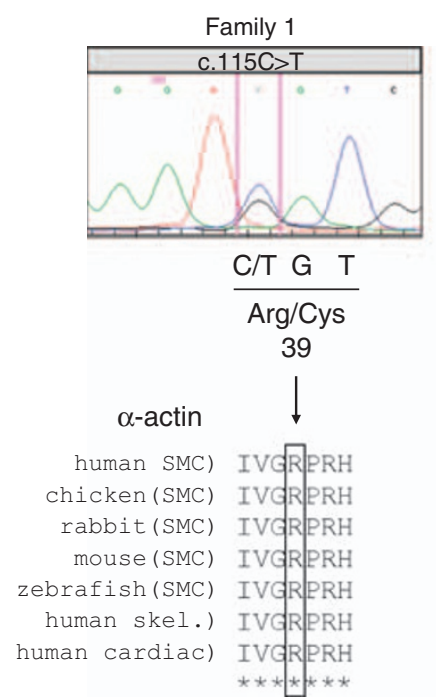

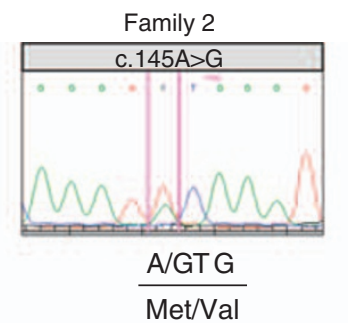

49

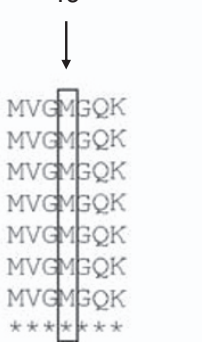

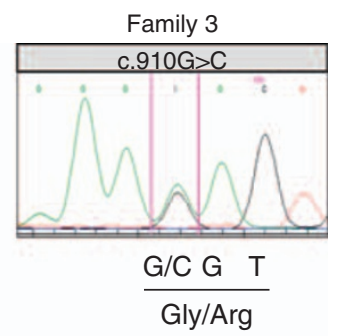

304

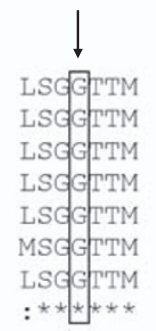

Figure 1 Three novel mutations in ACTA2 affecting evolutionarily conserved amino acids. Shown are sequencing electropherograms (upper panel) and the results from a multiple alignment of several smooth muscle cell (SMC), skeletal (skel.) or cardiac isoforms of $\alpha$-actin. The mutation nomenclature refers to GenBank reference sequenes NM_001613.2 and NP_001604.1, respectively. 
Table 1 Location of all reported ACTA2 mutations and vascular phenotypes observed in heterozygous mutation carriers, summarized from the studies by Guo et al, ${ }^{6}$ Morisaki et al $^{17}$ and the present analysis

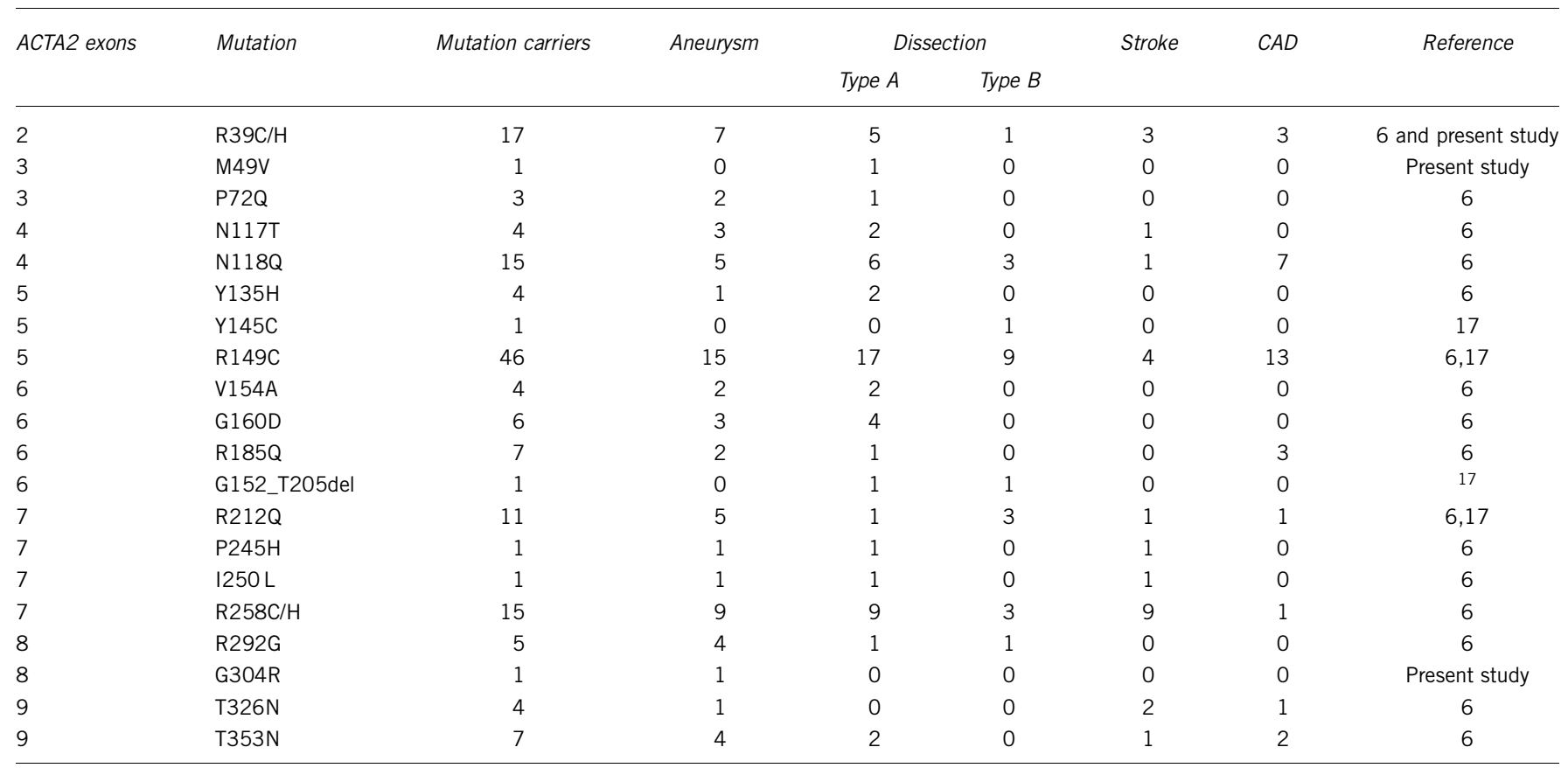

Family 1 (p.R39C)

Family 2 (p.M49V)

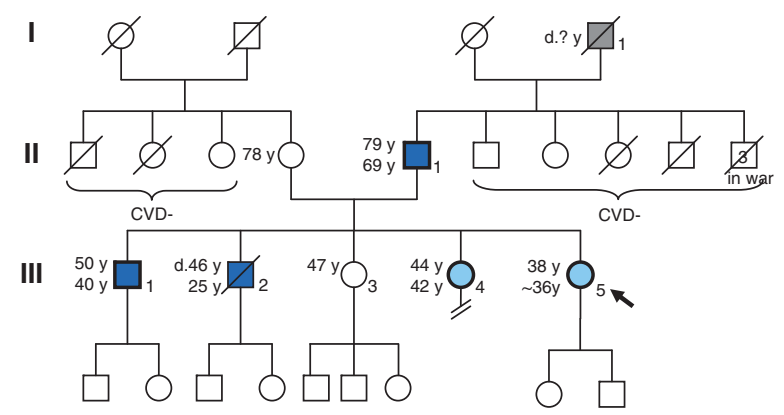

I

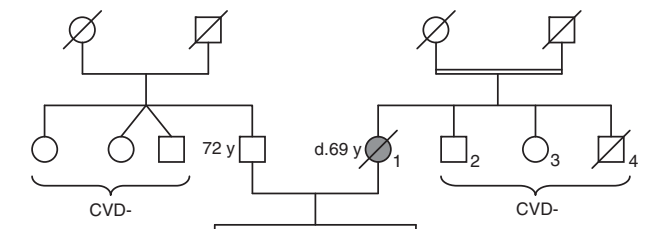

II

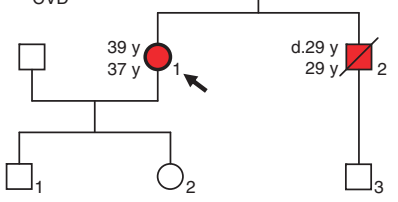

Family 3 (p.G304R)

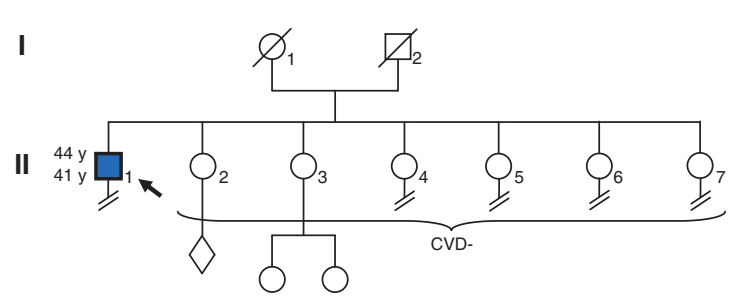

$\square$ carrier status unknown

$\square$ carrier of a mutation

$\square$ aortic aneurysm

$\square$ mild aortic dilatation and insufficiency

aortic dissection

sudden unexplained death

Figure 2 Family trees showing the segregation and clinical manifestations of the three novel ACTA2 mutations. Circles, squares and diamonds indicate women, men and offspring of disclosed sex, respectively. The current age (upper number) and the age at diagnosis of TAAD (lower number) are given and ' $d$ '. indicates age at death. Note that unaffected family members have not undergone genetic testing. CVD-: no known cardiovascular disease reported in these individuals as well as their offspring $(n=27)$.

sister of the index patient (patient III.3) was reported to be unaffected and declined genetic counselling and testing. None of the affected individuals in this family showed syndromic features consistent with Marfan, Ehlers-Danlos or Loeys-Dietz syndromes. Detailed evaluation of the family history (based on clinical history in the family members and recent clinical cardiologic and neurologic evaluation in the probands) did not reveal any case of premature stroke or CAD.
Family 2 (M49V). The mutation was revealed in a female patient who presented with acute aortic dissection (Stanford type A) at the age of 37 years (patient II.1, Figure 2). She previously had two uneventful pregnancies and deliveries, and reported to have been entirely healthy up to this point. Her brother had died at the age of 29 years from acute aortic dissection. Both siblings did not have syndromic features suggestive of Marfan, Ehlers-Danlos or Loeys-Dietz 

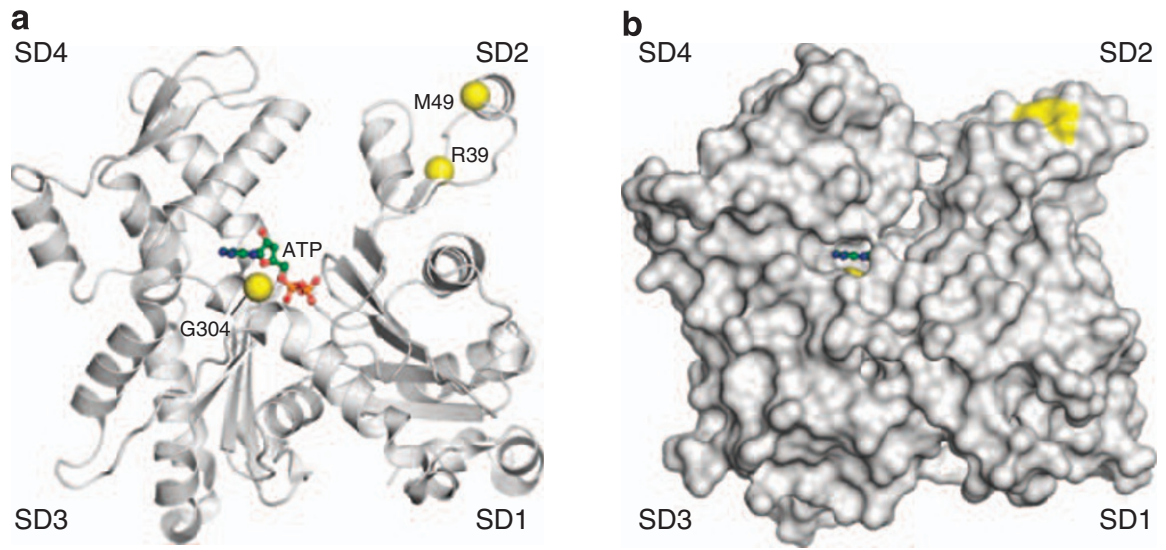

Figure 3 Localisation of mutated amino acids in a protein model of actin. The mutations were plotted onto a model of rabbit $\alpha$-actin (PDB code $1 \mathrm{~J} 6 \mathrm{Z}$ ) ${ }^{10}$. The numbering of the amino acids refers to the human reference sequence (NP_001604.1). SD1-4: subdomains 1-4 of the actin monomer. (a) Ribbon plot. The positions of the mutated amino acids presented in this work are marked by yellow spheres. (b) Solvent accessible surface. Note that residue G304 is buried within the ATP-binding pocket and that both R39 and M49 colocate on the surface of the D-loop.

syndrome. Their mother who suffered from gradually developing spastic paraplegia for over 20 years and cancer died suddenly at the age of 69 years in connection with a massive drop in blood pressure. No other family members were reported with known TAAD, premature $\mathrm{CAD}$ or stroke.

Family 3 (p.G304R). The mutation was identified in a male patient without syndromic features who developed a thoracic aortic aneurysm at the age of 41 years (patient II.1, Figure 2). His six sisters were reported to have no known cardiovascular disease and were not interested in genetic counselling or testing. His parents died early, presumably because of chronic abuse of alcohol. Additional information was not available from this family.

Positions of the mutations in a protein model of $\alpha$-actin In order to find clues to the primary molecular defect, the three mutations were mapped onto a three-dimensional structure of rabbit $\alpha$-actin. Two of the three novel mutations were found to affect residues within $(\mathrm{M} 49 \mathrm{~V})$ or adjacent to (R39C) the DNAse-I-binding loop (D-loop) within subdomain 2 (SD2) of $\alpha$-actin (Figure 3), which, upon cleavage of ATP, undergoes conformational changes that are believed to be critical for actin monomer interaction. ${ }^{10,15}$ Another missense mutation at position 39 has already been described in the literature $(\mathrm{R} 39 \mathrm{H}$, Table 1$)$ and has been associated with TAAD ${ }^{2}$ as well as with premature stroke and CAD. ${ }^{2}$ The third mutation affects residue Gly 304, which is buried in a hydrophobic pocket of the interdomain cleft known to bind ATP. ${ }^{16}$ Collectively, the positional data suggests that all three mutations could interfere with normal formation of actin filaments. Furthermore, the finding that residues Arg 39 and Met 49 map to an exposed loop of SD2 indicates that the mutations R39C and M49V may directly impact on the interaction with myosin or regulatory actin-binding proteins. These two mutations may therefore reduce the motile force generated by SMCs more directly than through a paucity of actin filaments.

\section{DISCUSSION}

\section{Genetic epidemiology of TAAD linked to ACTA2}

Since the first description of ACTA2 mutations involved in familial TAAD appeared in $2007,,^{2}$ a total of 19 mutations in this gene have been reported (see Table 1). ACTA2 mutations were found in 14-21\% of familial cases, but only $2.5-3.8 \%$ of sporadic TAAD. ${ }^{2,17}$ In this analysis, we detected mutations in ACTA2 in 3 out of 40 German TAAD patients $(7.5 \%)$. This detection rate (likely) reflects the mean detection rate in an unselected TAAD cohort, after exclusion of mutations in the FBN1 and TGFBR2 genes.

We identified three missense mutations in ACTA2 residing in highly conserved regions. This goes along with the fact that the mutations described so far were also almost exclusively missense mutations, except for one splice site mutation resulting in skipping of exon $6 .{ }^{2,6,17}$ The three mutations presented in this work add to the single mutations previously reported for exons 2,3 and 8 (Table 1) and therefore underpin the role of the $\mathrm{N}$ - and C-terminal portions of actin. Collectively, the genetic findings in the published cohorts suggest that routine genetic testing in TAAD should address all coding exons of ACTA2.

\section{Possible pathogenetic mechanisms}

The data presented in this work strongly suggest that the newly identified mutations may be pathogenetically relevant. Although functional data on the mutant proteins is not yet available, some conclusions on possible molecular consequences can be drawn on the basis of the position of the affected amino acids in a structural model. For example, G304R replaces an unpolar by a positively charged amino-acid residue in the hydrophobic pocket that binds to the adenine moiety of ATP. Moreover, the substitution of glycine by arginine introduces a side chain that is believed to sterically hinder actin-ATP interaction. Therefore, G304R, like R185Q described by Guo et $a l^{6}$ most likely perturbs ATP binding and/or hydrolysis. As the conformation of actin depends on the state of the bound nucleotide, ${ }^{18}$ such an alteration of ATP-binding likely translates into structural changes that may impair both fibrous-actin (F-actin) stability and actin-myosin interaction. Altered assembly and/or stability of actin filaments are also likely consequences of the two other mutations reported here. M49V affects the tip of the D-loop. By adopting an extended conformation, the D-loop contributes to the flattening of the molecule, which is thought to be essential for the formation of stable helical F-actin. ${ }^{19}$ A critical role may also be postulated for the arginine residue affected by the mutation R39C, which colocates with Met 49 on the surface of SD2 (Figure 3). A perturbation of the F-actin stability is presumed to lead to a paucity of actin filaments, such as that observed in SMCs from carriers of the R118Q and T353N mutations. ${ }^{2}$ Diminished steady-state levels of actin could also be 
because of efficient degradation of mutant globular actin by cellular surveillance systems, such as the ubiquitin proteasome system, leading to a functional null-allele and presumably, haplo-insufficiency. In this context it is worth noting that Guo et al ${ }^{2}$ suggested that ACTA2 mutations usually have a dominant negative effect and that mutations resulting in null-alleles may be inherited recessively. Clearly, both scenarios are likely to diminish the contractile power of the SMCs, which is thought to trigger compensatory remodelling of the tunica media, resulting in a stiff aorta prone to dilation and dissection. This remodelling process involves dysregulated SMC proliferation similar to the situation observed in TAAD patients carrying MYH11 mutations. ${ }^{5}$ In smaller vessels (eg coronary or cerebral arteries), focal SMC hyperplasia may lead to stenosis, which predisposes patients to occlusive conditions. ${ }^{6}$

\section{Clinical implications}

The finding of $\mathrm{R} 39 \mathrm{C}$, in addition to $\mathrm{R} 39 \mathrm{H},{ }^{6}$ further underpins the role of subdomain 2 of the actin protein. In family 1 , the mutation was linked to a variable vascular phenotype, ranging from mild aortic dilation and insufficiency in a woman aged 44 years to overt aortic aneurysm requiring surgical repair in a 25 -year-old male patient (suspected to carry R39C). Although R39H was found to be associated with type A dissections in two families and with type B dissections in one family, dissections were not observed in the R39C family reported here. Collectively, the data available so far indicate that the outcome of a substitution at Arg 39 is highly variable, ranging from no symptoms in the 7 th decade of life to sudden death before the age of 25 years (Table 1).

In addition to increased risk of aortic aneurysms and dissections, ACTA2 mutations have also been implicated in the development of premature $\mathrm{CAD}$ and stroke, ${ }^{6}$ and have been associated in some families with livedo reticularis, a skin discoloration because of constriction or occlusion of deep dermal capillaries. ${ }^{2}$ Yet, in the three patients harbouring ACTA2 mutations in this study, as well as in their family members, we did not find any case of premature CAD, stroke or livedo reticularis. Guo et al ${ }^{6}$ suggested that the different vascular diseases, for example, TAAD vs CAD or stroke, may be associated with specific ACTA2 missense mutations: mutations affecting residue 258 were primarily associated with stroke, whereas p.R118Q and p.R149C lead primarily to CAD. Interestingly, the $\mathrm{R} 39 \mathrm{H}$ mutation has also been implicated in premature stroke (before the age of 20). ${ }^{6}$ In this work, a different amino acid exchange in this latter residue (R39C) was observed in several family members affected by aortic aneurysm, but not by stroke or CAD. The number of mutation carriers investigated so far is certainly far too small to conclude that R39C, unlike R39H, exclusively predisposes to TAAD and not to CAD or stroke. The results by Guo et al rather suggest that the substitution of Arg 39 leads to a general risk of occlusive arterial disease that is not secondary to aortic dissection or aortic surgical repair. Therefore, all mutation carriers and their offspring should be closely monitored by clinical means and predictive genetic testing may be discussed for asymptomatic family members. Regarding the possible clinical consequences of the M49V mutation, our data suggest a risk of aortic dissection within the first four decades of life. Taking into account that Met 49 locates in the vicinity of Arg 39, one might argue that M49V can predispose to $\mathrm{CAD}$ and stroke (Figure 3). The third mutation presented in this work, G304R, affects a protein region that has been linked to a high frequency of CAD. ${ }^{6}$ Additional studies that include a higher number of mutation carriers are warranted to further elucidate the full phenotypic spectrum associated with these mutations.

In conclusion, we confirm the role of ACTA2 mutations in nonsyndromic TAAD. Particularly in young patients with aortic disease that do not fulfil the diagnostic criteria for MFS, ACTA2 analysis is likely to have an important place in routine diagnostics. ${ }^{20}$ ACTA2 should therefore definitely be included in the genes investigated by future resequencing chips for syndromic and nonsyndromic TAAD.

\section{CONFLICT OF INTEREST}

The authors declare no conflict of interest.

\section{ACKNOWLEDGEMENTS}

We would like to thank Joanne Davies, Janine Haremza, Melanie Müller and Kirsten Rackebrandt for technical assistance and the patients for participating in this study.

\section{WEB RESOURCES}

URLs for data presented herein are as follows:

GenBank, http://www.ncbi.nih.gov/Genbank/index.html RCSB Protein Data Bank, http://www.rcsb.org/pdb/Welcome.do OMIM, http://www.ncbi.nlm.nih.gov/Omim/

HGVS, http://www.hgvs.org/

PANTHER, http://www.pantherdb.org/tools/csnpScoreForm.jsp

PolyPhen, http://genetics.bwh.harvard.edu/pph/

ClustalW2, http://www.ebi.ac.uk/Tools/clustalw2/index.html

1 Milewicz DM, Guo DC, Tran-Fadulu V et al: Genetic basis of thoracic aortic aneurysms and dissections: focus on smooth muscle cell contractile dysfunction. Annu Rev Genomics Hum Genet 2008; 9: 283-302.

2 Guo DC, Pannu H, Tran-Fadulu V et al: Mutations in smooth muscle alpha-actin (ACTA2) lead to thoracic aortic aneurysms and dissections. Nat Genet 2007; 39: 1488-1493.

3 Zhu L, Vranckx R, Khau Van Kien P et al: Mutations in myosin heavy chain 11 cause a syndrome associating thoracic aortic aneurysm/aortic dissection and patent ductus arteriosus. Nat Genet 2006; 38: 343-349.

4 Dillon PF, Aksoy MO, Driska SP, Murphy RA: Myosin phosphorylation and the cross-bridge cycle in arterial smooth muscle. Science 1981; 211: 495-497.

5 Pannu H, Tran-Fadulu V, Papke CL et al: MYH11 mutations result in a distinct vascular pathology driven by insulin-like growth factor 1 and angiotensin II. Hum Mol Genet 2007; 16: 2453-2462.

6 Guo DC, Papke CL, Tran-Fadulu $V$ et al: Mutations in smooth muscle alpha-actin (ACTA2) cause coronary artery disease, stroke, and Moyamoya disease, along with thoracic aortic disease. Am J Hum Genet 2009; 84: 617-627.

7 Hoffman JI, Kaplan S: The incidence of congenital heart disease. J Am Coll Cardiol 2002; 39: 1890-1900.

8 Miller SA, Dykes DD, Polesky HF: A simple salting out procedure for extracting DNA from human nucleated cells. Nucleic Acids Res 1988; 16: 1215.

9 Waldmuller S, Muller M, Warnecke $\mathrm{H}$ et al: Genetic testing in patients with aortic aneurysms/dissections: a novel genotype/phenotype correlation? Eur J Cardiothorac Surg 2007; 31: 970-975.

10 Otterbein LR, Graceffa P, Dominguez R: The crystal structure of uncomplexed actin in the ADP state. Science 2001; 293: 708-711.

11 Berman HM, Westbrook J, Feng Z et al: The Protein Data Bank. Nucleic Acids Res 2000; 28: 235-242.

12 Schrodinger L: The PyMOL Molecular Graphics System, Version 1.3, 2010.

13 Laing NG, Dye DE, Wallgren-Pettersson C et al: Mutations and polymorphisms of the skeletal muscle alpha-actin gene (ACTA1). Hum Mutat 2009; 30: 1267-1277.

14 Hung RM, Yoon G, Hawkins CE, Halliday W, Biggar D, Vajsar J: Cap myopathy caused by a mutation of the skeletal alpha-actin gene ACTA1. Neuromuscul Disord 2010; 20: 238-240.

15 Holmes KC, Popp D, Gebhard W, Kabsch W: Atomic model of the actin filament. Nature 1990; 347: 44-49.

16 Mannherz HG: Crystallization of actin in complex with actin-binding proteins. J Biol Chem 1992; 267: 11661-11664.

17 Morisaki $\mathrm{H}$, Akutsu $\mathrm{K}$, Ogino $\mathrm{H}$ et al: Mutation of ACTA2 gene as an important cause of familial and nonfamilial nonsyndromatic thoracic aortic aneurysm and/or dissection (TAAD). Hum Mutat 2009; 30: 1406-1411.

18 Page R, Lindberg U, Schutt CE: Domain motions in actin. J Mol Biol 1998; 280: 463-474.

19 Oda T, Iwasa M, Aihara T, Maeda Y, Narita A: The nature of the globular- to fibrous-actin transition. Nature 2009; 457: 441-445.

20 Akutsu K, Morisaki H, Okajima T et al: Genetic analysis of young adult patients with aortic disease not fulfilling the diagnostic criteria for Marfan syndrome. Circ J 2010; 74: 990-997. 